\title{
Predictores de la competencia léxica del futuro profesorado
}

\author{
Predictors of the lexical competence of future teachers
}

\author{
INMACULADA CLOTILDE SANTOS-DÍAZ ${ }^{a}$ \\ ESTER TRIGO-IBÁÑEZ ${ }^{\mathrm{b}}$ \\ MANUEL FRANCISCO ROMERO-OLIVA ${ }^{\mathrm{b}}$ \\ ${ }^{a}$ Universidad de Málaga, Facultad de Ciencias de la Educación. España. \\ Correo electrónico: santosdiaz@uma.es \\ ${ }^{b}$ Universidad de Cádiz, Facultad de Ciencias de la Educación, España. \\ Correos electrónicos: ester.trigo@uca.es, manuelfrancisco.romero@uca.es
}

El desarrollo de la competencia léxica es un factor clave en todas las etapas educativas donde el docente no solo actúa como guía sino también como modelo lingüístico. El objetivo de esta investigación es conocer cuáles son los factores que se relacionan con el caudal léxico del futuro profesorado. Para recabar y procesar los datos se toman como referencia las directrices metodológicas de una disciplina afianzada, la disponibilidad léxica. La muestra está formada por 171 estudiantes del Máster de Profesorado de la Universidad de Málaga. Los principales predictores del léxico en espańol hallados en el análisis de regresión lineal son el léxico en lengua extranjera, la especialidad del máster, la capacidad de identificación de tecnicismos y los hábitos de lectura. Estos hallazgos abren nuevas vías de investigación en el ámbito de la disponibilidad léxica e invitan a reflexionar sobre el fomento de programas específicos de mejora de la competencia lingüística.

Palabras claves: Disponibilidad Léxica, Hábitos Lectores, Formación del Profesorado, Sociolingüística, Vocabulario.

The development of lexical competence is a key factor in all educational stages where the teacher not only acts as a guide but also as a linguistic model. The objective of this research is to know which are the factors that are related to the lexical flow of future teachers. To collect and process the data, the methodological guidelines of an established discipline, lexical availability, are taken as a reference. The sample is made up of 171 students from the master's degree in Teaching at the University of Malaga. The main predictors of the Spanish lexicon found in the linear regression analysis are the foreign language lexicon, the master's specialty, the ability to identify technicalities and reading habits. These findings open new avenues of research in the field of lexical availability and invite us to reflect on the promotion of specific programs to improve linguistic competence.

Key words: Lexica Availability, Reading Habits, Teacher Training; Sociolinguistics, Vocabulary. 


\section{INTRODUCCIÓN}

Con el inicio de la segunda mitad del siglo pasado, un grupo de lingüistas franceses (Gougenheim, Michéa, Rivenc y Sauvageot 1956, 1964) se plantea compilar un vocabulario de base para la enseñanza del francés como segunda lengua. La necesidad surge por la urgencia de que tanto los habitantes de la antigua Unión Francesa como los inmigrantes que llegaban a Francia pudieran aprender el idioma con cierta rapidez. En una primera fase, se realiza una selección del léxico a partir de los listados de vocabulario frecuente. Sin embargo, los problemas no tardan en aflorar, pues las palabras más frecuentes distaban mucho de ser las más utilizadas por los hablantes: era preciso contar, en esos repertorios léxicos, con palabras con carga semántica, es decir, sustantivos, adjetivos y verbos.

Es así como se esbozan las claves de la disponibilidad léxica, basadas en la concepción de que en toda lengua existe un amplio conjunto de palabras de alto contenido semántico específico cuya aparición se relaciona con el tema del discurso. Para obtener el léxico disponible debe combinarse frecuencia y orden de aparición. De este modo, comienzan a aplicarse pruebas de léxico asociativo en las que los participantes deben actualizar, en un tiempo determinado, todas las palabras que vengan a su mente dado un estímulo o centro de interés. Michéa (1953), al referirse al vocabulario disponible, señala lo siguiente:

Mais que faut-il entendre par 'vocabulaire disponible'? Un mot disponible est un mot qui, sans être particulièrement fréquent, est cependant toujours prêt à être employé et se présente immédiatement et naturellement à l'esprit au moment où l'on en a besoin. C'est un mot qui, faisant partie d'associations d'idées usuelles, existe en puissance chez le sujet parlant, dès que ces associations entrent en jeu. L'existence d'un vocabulaire disponible n'est pas une vue de l'esprit. Il suffit de s'observer soi-même lorsqu'on parle et surtout lorsqu' on écrit pour se rendre compte que, souvent, plusieurs termes entrent en concurrence au moment de l'expression. Un seul est employé. Les autres ont simplement marqué discrètement leur présence (340).

A partir de estos primeros estudios, Mackey (1971) desarrolla un proyecto que pretendía comparar el léxico de Francia con el de las regiones francófonas de Canadá. Sin embargo, "el verdadero renacimiento de los viejos estudios surgió en tierras americanas hispanohablantes para poco después extenderse exitosamente por España" (López Morales 2011: 15). Progresivamente, los trabajos de disponibilidad léxica han ido avanzando epistemológicamente y no han faltado oportunidades para reflexionar sobre las cuestiones metodológicas de la disciplina (Paredes García 2012; López González 2014). Así, desde el punto de vista estadístico, se han desarrollado enfoques inéditos en esta disciplina, aplicando novedosos índices matemáticos (Ávila Muñoz y Sánchez Sáez 2014; Callealta Barroso y Gallego Gallego 2016), realizando un análisis factorial (Trigo Ibánez, Romero Oliva y Santos Díaz 2019a), trabajando con minería de datos mediante el uso de árboles Chaid 
(Trigo Ibáñez, Romero Oliva y Santos Díaz 2020) o utilizando el análisis de regresión lineal (Ferreira, Garrido Moscoso y Guerra Rivera 2019).

En el ámbito hispánico, la extraordinaria expansión de esta línea de investigación se atribuye a la existencia de un Proyecto Panhispánico de Disponibilidad Léxica (en adelante, PPHDL), dirigido por el profesor Humberto López Morales. La información acerca de los avances de este proyecto puede encontrarse en la web: http://www.dispolex.com, gestionada por José Antonio Bartol Hernández y Natividad Hernández Muñoz, de la Universidad de Salamanca. Esta web supone un foro de encuentro entre investigadores del ámbito y una excelente base de datos aglutinadora de las investigaciones integradas en esta línea.

En el PPHDL se toman como referencia muestras de población formadas por alumnado del último curso de bachillerato al considerarlos hablantes maduros que no han visto aún su léxico especializado por estudios universitarios. Se consignan, al menos, 16 centros de interés -1) Partes del cuerpo humano; 2) La ropa; 3) Partes de la casa (sin los muebles); 4) Los muebles de la casa; 5) Comidas y bebidas; 6) Objetos colocados en la mesa para la comida; 7) La cocina y sus utensilios; 8) La escuela: muebles y materiales; 9) Iluminación, calefacción y medios para airear un recinto; 10) La ciudad; 11) El campo; 12) Medios de transporte; 13) Trabajos del campo y del jardin; 14) Los animales; 15) Juegos y distracciones; 16) Profesiones y oficios - y cuatro variables extralingüísticas -sexo, tipo de centro educativo, localización geográfica y nivel sociocultural-. Además de estos acuerdos, también se han establecido unas pautas de edición comunes (Samper Padilla 1998; Samper Padilla, Bellón Fernández y Samper Hernández 2003), que han posibilitado la realización de avances conjuntos y de fructíferas comparaciones diatópicas.

A partir de este proyecto, han proliferado los trabajos aplicados a diversas disciplinas, utilizando diferentes poblaciones y rangos etarios (De la Maya Retamar y López Pérez 2020; Herranz Llácer 2019; López Morales y Trigo Ibáñez 2019; Zambrano Matamala, Rojas Díaz y Salcedo Lagos 2019). De todas las líneas de aplicación adquiere especial importancia la perspectiva de la enseñanza de lenguas, donde se encuentran trabajos centrados en las bases cognitivas para la adquisición léxica (Hernández Muñoz y Tomé Cornejo 2017), la selección del léxico adecuado (Santos Díaz 2017a; Rubio Lastra 2020); la enseñanza de ELE (Sánchez-Saus Laserna 2016; Hidalgo Gallardo 2019), la ortografía (Paredes García 1999; Trigo Ibáñez, Romero Oliva y Santos Díaz 2018, 2019a, 2020; Santos Díaz, Trigo Ibáñez y Romero Oliva 2019), la enseñanza del léxico, en general (Santos Díaz, Trigo Ibáñez y Romero Oliva 2020) o el dialectal, en particular (Trigo Ibáńez, Romero Oliva y Santos Díaz 2019b; Trigo Ibáñez, Santos Díaz y Romero Oliva 2021; Galloso Camacho y Martín Camacho 2019). Tampoco faltan los estudios centrados en la enseñanza de segundas lenguas: francés (Mora Ramos 2014; De la Maya Retamar 2015; De la Maya Retamar y Mora Ramos 2019) o inglés (De la maya Retamar y López Pérez 2020, 2021; Jiménez Catalán 2014; Ferreira 2006; Ferreira y Echeverría 2010-2011).

Si bien el PPHDL seleccionaba informantes preuniversitarios, actualmente estos estudios se han extendido - en uno y otro hemisferio- a grupos poblacionales diversos. De todos ellos, por su implicación con la enseńanza, resulta interesante estudiar con mayor 
detenimiento el formado por docentes en formación. Estos trabajos recogen las siguientes líneas temáticas:

- La evaluación de la competencia léxica en lengua materna (Rojas Díaz, Zambrano Matamala y Salcedo Lagos 2017; Herranz Llácer 2018; Herranz Llácer y Marcos Calvo 2019; Castillo Fadić y Sologuren Insúa 2020; Santos Díaz 2020), o en lengua extranjera (Santos Díaz 2017c; Quintanilla Espinoza y Salcedo Lagos 2019; De la Maya Retamar y López Pérez 2019, 2020; Santos Díaz 2020);

- el manejo del léxico terminológico según aumenta la formación docente (Cerda Etchepare, Salcedo Lagos, Pérez Wilson y Marín Díaz 2017; Valenzuela Castellanos, Pérez Villalobos, Bustos y Salcedo Lagos 2018; Marcos Calvo y Herranz Llácer 2019; Juárez Calvillo 2019);

- la evaluación de la competencia ortográfica en lengua materna y extranjera (Santos Díaz, Trigo Ibáñez y Romero Oliva 2019);

- la toma de decisiones didácticas a partir de los hallazgos encontrados (Rojas Díaz, Zambrano Matamala y Salcedo Lagos 2019; Santos Díaz, Trigo Ibáñez y Romero Oliva 2020);

- la selección léxica para la enseñanza de lenguas (Santos Díaz 2017a); o

- la relación entre el hábito lector y la competencia léxica (Santos Díaz 2017b).

Como se aprecia, esta línea de investigación resulta altamente interesante, pues la evaluación de la competencia léxica de los futuros docentes, su concepción sobre determinados temas o el afianzamiento de la terminología propia de los estudios cursados será crucial, no solo para proponer estrategias encaminadas a la mejora de la formación inicial, sino también para reflexionar sobre los materiales didácticos existentes y trazar líneas de prospección didáctica tanto para áreas lingüísticas como no lingüísticas.

En este sentido, esta investigación persigue determinar cuáles son las variables más influyentes en la competencia léxica de los docentes en formación de la Universidad de Málaga a partir de un análisis de regresión múltiple.

\section{Metodología}

\subsection{Participantes}

En esta investigación han participado 171 estudiantes matriculados en el curso 2010-2011 en 10 especialidades del Máster en Profesorado de Educación Secundaria Obligatoria, Bachillerato, Formación Profesional y enseñanza de idiomas de la Universidad de Málaga. 54 son hombres y 117 son mujeres. Su edad oscila entre los 22 y los 49 años, siendo 28,73 el valor etario medio. Para acceder a estos estudios, los informantes han debido acreditar un nivel mínimo de B1 en una lengua extranjera (las elegidas han sido inglés o 
francés) según el Marco Europeo Común de Referencia de las Lenguas. A priori, se cuenta con un error de muestreo del 8,5\%, calculado a partir de la relación entre los 171 elementos de la muestra y los 385 de la población total. Sin embargo, pese a que la población $(\mathrm{N}=$ 385), correspondiente a los estudiantes matriculados, no es coincidente con la muestra $(\mathrm{n}=171)$, constituida por los estudiantes presentes, se considera que la muestra tiene un carácter censal al estar compuesta por informantes representativos y válidos (Dörnyei y Csizér 2012). De esta forma, como muestra la Tabla 1 , los cuestionarios fueron aplicados al alumnado accesible, estratificado posteriormente según las especialidades cursadas.

Tabla 1. Distribución de los informantes que conforman la muestra de estudio por especialidad

\begin{tabular}{|l|c|}
\hline Especialidad del máster & Participantes \\
\hline Dibujo, Imagen y Artes Plásticas & 11 \\
\hline Formación y Orientación Laboral & 7 \\
\hline Ciencias Sociales, Geografía e Historia & 17 \\
\hline Hostelería y Turismo & 10 \\
\hline Lengua extranjera (Inglés y Francés) & 38 \\
\hline Lengua Castellana y Literatura, Latín y Griego & 19 \\
\hline Música & 9 \\
\hline Orientación Educativa & 10 \\
\hline Tecnología, Informática y Procesos Industriales & 40 \\
\hline Matemáticas & 10 \\
\hline Total & 171 \\
\hline
\end{tabular}

Fuente: Elaboración propia.

Atendiendo al número de informantes y a la calidad de los datos recabados, la presente investigación cumple con los criterios de suficiencia y adecuación expuestos por Rodríguez Gómez, Gil Flores y García Jiménez (1996). En lo que respecta a los criterios éticos, se han contemplado factores claves como la confidencialidad de los datos, el anonimato y la transparencia a la hora de recoger y presentar los resultados.

\subsection{Instrumentos}

El acceso a los informantes se realizó cuidadosamente. Para ello fue necesario contar con un documento de negociación, entregado previamente a los miembros de la 
comisión académica de la titulación, docentes y estudiantes. Los datos extralingüísticos, que permitieron describir a cada informante, se recogieron a través de un cuestionario sociológico. Los lingüísticos, que facilitaron el conocimiento de la competencia léxica de los participantes, fueron obtenidos a partir de una prueba de disponibilidad léxica y otra prueba específica para la identificación de léxico especializado:

- Cuestionario sociológico. La encuesta (Santos Díaz 2020) se inicia con un apartado dedicado a recabar datos identificativos para conocer el grupo, el idioma en el que se realiza la prueba y la fecha exacta en que se completó el cuestionario. Seguidamente, aparecen 27 preguntas sociológicas para compilar la información relacionada con la formación y con el contacto con otras lenguas tanto del informante como de sus familiares. Además, se consignan ítems relacionados con el nivel económico del informante, su movilidad geográfica y con la densidad de sus relaciones. Al tratarse de cuestionarios anónimos, la codificación se realizó según el orden de recogida.

- Prueba específica para la identificación del léxico especializado -vid. Anexo-. Se ha tomado como referencia la investigación de Ávila Muñoz y Villena Ponsoda (2010). En ella, los informantes debían relacionar dos palabras con cada centro de interés en los dos idiomas en los que realizaba la prueba. En total se contaba con 18 palabras especializadas. Por ejemplo, en el centro de interés Partes del cuerpo bumano los tecnicismos que debían identificarse eran: escroto e isquion.

- Prueba de léxico disponible. Al final de cada cuestionario, los informantes podían encontrar 10 folios en blanco para realizar la prueba. Se trata del clásico test asociativo de los estudios de disponibilidad léxica. En esta ocasión, y dado que el léxico se ha se ha recogido en dos idiomas -inglés $(n=150)$ o francés $(n=21)$, según el idioma acreditado para acceder al máster-, en lugar de contar con los 16 centros de interés propios del PPHDL (vid. Introducción), se han incluido 9 solamente, 8 de ellos son tradicionales (Gougenheim et al. 1956, 1964): 1) Partes del cuerpo humano; 2) La ropa; 3) Comidas y bebidas; 4) La escuela (muebles y materiales); 5) La ciudad; 6) Medios de transporte; 7) Juegos y distracciones; 8) Profesiones y oficios. A estos se ha añadido un noveno, 9) Ordenadores e internet, debido a su relación con la profesión docente en la actualidad. Con la reducción del número de centros de interés se garantizó que la prueba tuviese una duración similar a la de los estudios del PPHDL, unos 40 minutos.

\subsection{Procedimiento}

Al recopilar el léxico se siguieron las directrices metodológicas más utilizadas en los estudios de disponibilidad léxica (López González 2014; Santos Díaz 2020), según las cuales cada informante escribe aquello que le viene a la mente dado un estímulo -en este caso, un centro de interés- durante dos minutos, sin prestar atención a la corrección ortográfica. Para su procesamiento, se incluyeron todas las palabras, independientemente de 
su conexión con el estímulo verbal -o centro de interés-con el fin de no perder información relevante tal y como recomiendan estudios anteriores (Ávila Muñoz y Villena Ponsoda 2010, 2011; Hernández Muñoz 2004). En cuanto a la lematización, se tuvieron en cuenta tanto las recomendaciones de Samper Padilla (1998) como criterios propios (Santos Díaz 2020). El índice de disponibilidad léxica se obtuvo a partir de la fórmula de López Chávez y Strasburguer Frías (1987), utilizando el programa Lexidisp (Moreno Fernández, Moreno Fernández y García de las Heras 1995).

El caudal léxico de los informantes resultante de esta prueba suele calcularse sumando el número total de palabras, ya sea por centro de interés o de forma global (Herranz Llácer 2018; Santos Díaz 2020). Sin embargo, este estudio toma como referencia el cálculo del Índice Corregido de Descentralización Léxica (en adelante, ICoDD) propuesto por Ávila Muñoz y Sánchez Sáez (2010) y utilizado en otros estudios como el realizado por Ávila Muñoz, Santos Díaz y Trigo Ibáńez (2020). Este índice tiene en cuenta las características de cada palabra aportada por los informantes con respecto a su centro de interés. En un primer momento, a cada palabra se le otorga un valor en función de su relación con el núcleo temático, distinguiendo entre: palabras nucleares (valor 1), asociaciones colectivas (valor 0,5 ), palabras derivadas (valor 0,25 ) y asociaciones individuales (valor 0 ). A continuación, se calcula la compatibilidad de los vocablos aplicando la teoría de los conjuntos difusos. Finalmente, se calcula la descentralidad restando 1 a la compatibilidad léxica mostrada por cada informante. El resultado es un parámetro que mide la capacidad de un informante de aportar vocabulario específico en un tema determinado. De esta forma, no solo se tiene en cuenta la cantidad de palabras que han actualizado los informantes sino también su pertinencia y especificidad ya que se presupone que son informantes capaces tanto de aportar palabras comunes y prototípicas del tema, como de aquellas más específicas.

Atendiendo a la prueba de léxico especializado - vid. Anexo-, sus resultados se mostraron a través del Pident definido como la capacidad individual de identificación de piezas léxicas especializadas (Ávila Muñoz y Villena Ponsoda 2010). Este índice se obtiene a partir de la suma de todas las respuestas correctas de los informantes, sin tener en cuenta el azar implícito en la prueba. Para hallar el índice corregido de identificación de tecnicismos (en adelante, ICIT), en este trabajo se han restado las respuestas incorrectas al total de respuestas correctas. A las correctas se les da el valor de 0,56 y a las incorrectas, de 0,70, calculado según la distribución de la probabilidad.

La información recopilada en la encuesta sociológica permitió establecer una serie de variables que, una vez codificadas y organizadas en un archivo en formato Excel, se importaron a una matriz de datos en el paquete estadístico SPSS (v. 22), lo que ha posibilitado la realización de análisis estadísticos bivariantes y multivariantes. En primera instancia se realiza un cribado de las variables que resultarán significativas a nivel bivariante. Tras este cribado, se lleva a cabo un análisis de regresión múltiple.

En concreto, el análisis de regresión múltiple explica la relación que ejerce un conjunto de variables independientes $\left(\mathrm{X}_{\mathrm{i}}\right)$ sobre una variable dependiente $(\mathrm{Y})$ (Calvo Gómez 1993). El objetivo de este modelo estadístico es doble: por una parte, predecir el 
factor funcional que relaciona las variables y, por otra parte, comprender las interrelaciones entre estas. Según Calvo Gómez (1993: 194), "el principio metodológico-estadístico de la Regresión Múltiple consiste en encontrar aquella función que mejor prediga $Y$, es decir, aquella que a partir de las variables independientes $\mathrm{X}$ menor error cuadrático de predicción cometa".

Para Albarrán Lozano, Guillén Estany y Ayuso Gutiérrez (2003) el modelo de regresión es concebido como la antesala de la interpretación de los resultados de investigaciones empíricas. El modelo de regresión lineal múltiple se rige en función del concepto de dependencia estocástica entre las variables. Por ello, este lleva incluido un término de perturbación aleatoria que intenta corregir ese valor azaroso.

En este trabajo se lleva a cabo un análisis de modelación lineal automatizada con el objetivo de crear un modelo estándar único que explique las relaciones entre campos. El modelo vincula la distribución aleatoria de la variable endógena con diferentes predictores lineales extraídos de las variables exógenas. En este tipo de análisis se incluyen todas las variables que cumplen determinados requisitos, ya que el resto de las variables se desestiman y no influyen en los resultados. El método seleccionado es el método forward o paso adelante en el que los regresores correlacionados significativamente con la variable dependiente se introducen uno a uno.

\section{Resultados}

La formación académica se dibuja como la variable más influyente en los sujetos. En este sentido, se parte de la hipótesis de que los hablantes que han realizado estudios ligados a algún área lingüística hayan profundizado en la lengua española y demuestren un léxico más descentralizado. A su vez, se incorporan las variables que en una fase anterior mostraron relaciones significativas a nivel bivariado: el número de libros que los estudiantes leen al año (Santos Díaz 2017b) y la clase social (Santos Díaz 2018).

La variable dependiente en este modelo es el ICoDD y las variables independientes seleccionadas son: el ICoDD en lengua extranjera, la especialidad del máster, la capacidad de identificación de tecnicismos y los hábitos de lectura. La clase social ocupa un papel secundario que aparece significativo al eliminar valores como el índice de descentralización léxica en español. Por tanto, en este análisis no aparece porque resulta poco significativa o redundante.

El modelo se explica en un 46,3 \% con un criterio de información de -925,457. El pronóstico observado - vid. Figura 1- muestra que, aunque la distribución de los valores no crea una línea de 45 grados, muestra la tendencia de que los datos se concentran en torno a la media dibujando una línea ascendente poco inclinada. 
Figura 1. Pronóstico por observado del ICoDD en lengua extranjera.

\section{Pronóstico por observado}

\section{Objetivo: ICoDD en español}

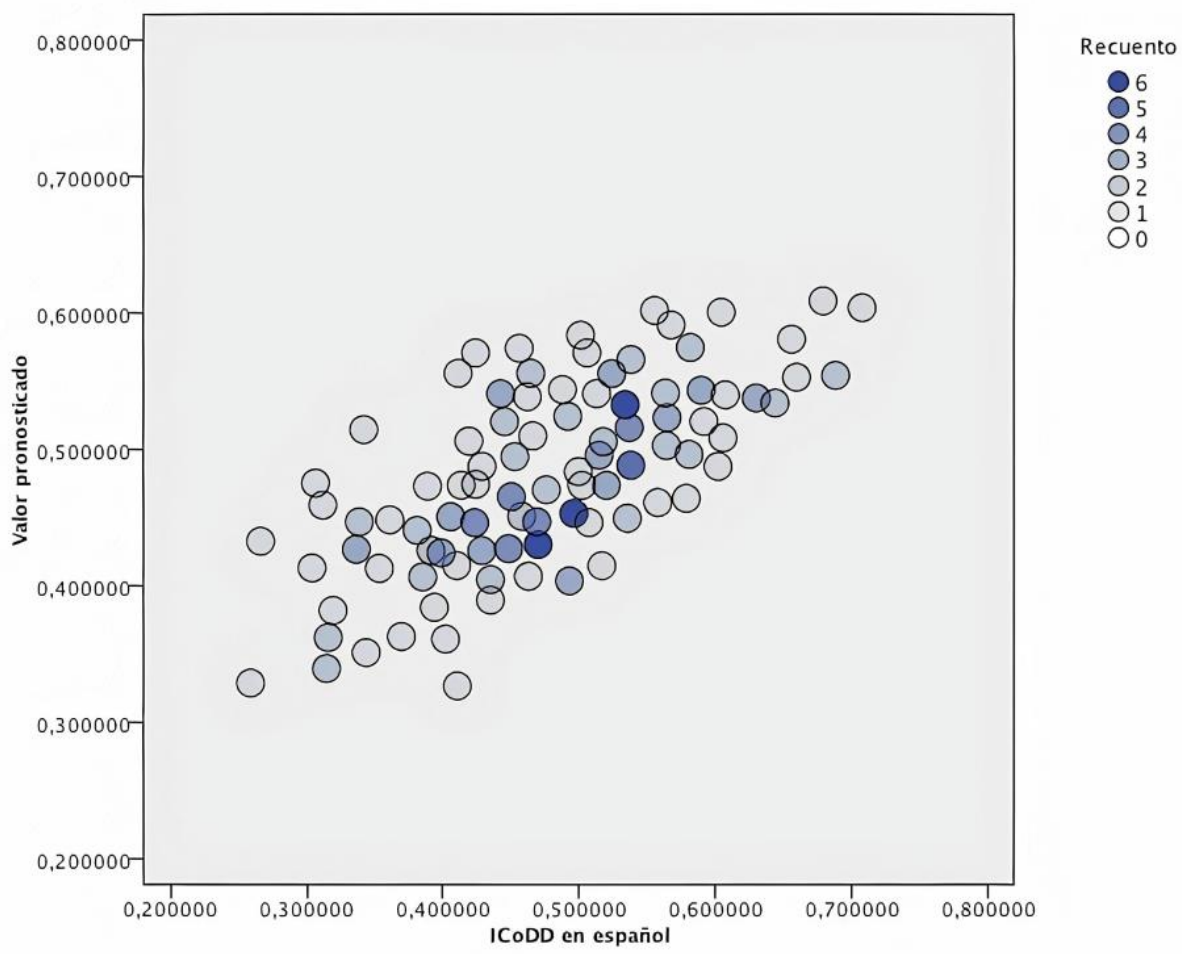


Por su parte, el histograma de los residuos estudentizados -vid. Figura 2-, demuestra que, en líneas generales, los residuos siguen una distribución normal, igual que la distribución típica $(1,003)$. No obstante, se destacan dos frecuencias de residuos $(-0,67$ y $0 ; 1$ y 1,33) alejadas de la línea de distribución normal.

Figura 2. Histograma de residuos estudentizados del ICoDD en lengua extranjera.

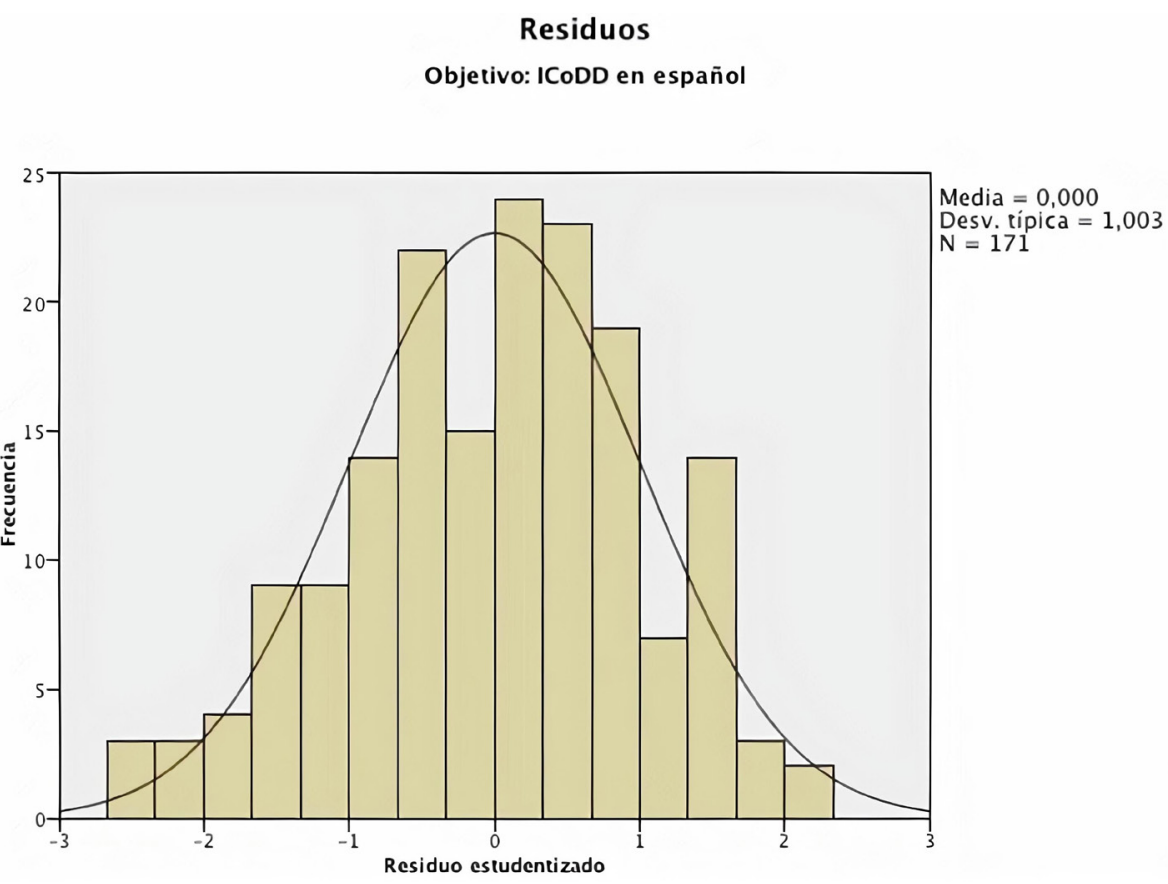

En cuanto a la importancia de cada variable en el modelo de regresión -vid. Tabla 2-, destaca el caudal léxico en lengua extranjera con un coeficiente Beta de 0,303 y una importancia de 0,545. En segundo lugar, se han establecido tres grupos según los resultados de las especialidades: el grupo 1 está formado por la especialidad de Matemáticas y tiene una incidencia negativa sobre el caudal léxico en lengua española; el grupo 2 lo conforman las especialidades de Lengua Extranjera (Inglés y Francés), Lengua Española, Latín y Griego y Música y tienen una influencia positiva con un nivel de significancia de 0,000; el tercer grupo está compuesto por las especialidades de Geografía e Historia, Dibujo, Orientación, FOL, Hostelería y Turismo y TIPI y no ejercen ningún efecto sobre el léxico disponible. 
Tabla 2. Datos estadísticos sobre el modelo de regresión lineal del caudal léxico en español

\begin{tabular}{|c|c|c|c|c|c|c|c|}
\hline & \multirow[b]{2}{*}{$\begin{array}{c}\text { Coeficiente } \\
\text { Beta }\end{array}$} & \multirow[b]{2}{*}{$\begin{array}{l}\text { Error } \\
\text { típico }\end{array}$} & \multirow[b]{2}{*}{$\mathbf{t}$} & \multirow[b]{2}{*}{ Significancia } & \multicolumn{2}{|c|}{ Nivel de confianza } & \multirow[t]{2}{*}{ Importancia } \\
\hline & & & & & Inferior & Superior & \\
\hline Intersección & 0,275 & 0,029 & 9,608 & 0,000 & 0,218 & 0,332 & \\
\hline $\begin{array}{l}\text { ICODD } \\
\text { en lengua } \\
\text { extranjera }\end{array}$ & 0,303 & 0,055 & 5,541 & 0,000 & 0,195 & 0,410 & 0,545 \\
\hline $\begin{array}{l}\text { Especialidad. } \\
\text { Grupo } 1\end{array}$ & $-0,050$ & 0,023 & $-2,135$ & 0,034 & $-0,134$ & $-0,004$ & 0,347 \\
\hline $\begin{array}{l}\text { Especialidad. } \\
\text { Grupo } 2\end{array}$ & 0,050 & 0,014 & 3,695 & 0,000 & 0,023 & 0,077 & 0,347 \\
\hline $\begin{array}{l}\text { Especialidad. } \\
\text { Grupo } 3\end{array}$ & $0,000_{\mathrm{a}}{ }^{1}$ & & & & & & 0,347 \\
\hline $\begin{array}{l}\text { ICIT en } \\
\text { español }\end{array}$ & 0,006 & 0,003 & 1,985 & 0,049 & 0,000 & 0,012 & 0,070 \\
\hline $\begin{array}{l}\text { Hábitos de } \\
\text { lectura }\end{array}$ & 0,008 & 0,006 & 1,446 & 0,053 & $-0,003$ & 0,020 & 0,037 \\
\hline
\end{tabular}

${ }^{1}$ Este coeficiente está establecido en cero porque es redundante.

Por orden de relevancia sigue la variable de identificación del léxico especializado con una importancia de 0,070 y los hábitos de lectura con 0,037. Ambas variables tienen una influencia positiva en el caudal léxico y no se distinguen subconjuntos. La variable referida a la lectura presenta un nivel de significancia superior al 0,5 razón por la cual en el modelo no se incluye la media estimada. 


\subsection{Medias estimadas}

En la Figura 3 se recogen las medias estimadas del ICoDD en español y el ICoDD en lengua extranjera y se evidencia que la relación entre ambas es significativamente positiva. Por consiguiente, se podría decir que aquellos informantes con un mayor caudal léxico en español lo tendrán de forma recíproca en inglés o francés. Asimismo, el ICoDD en lengua extranjera podría servir como variable intermedia, ya que transmite la importancia de las especialidades del máster que requieren estudios de lengua extranjera que, a su vez, son las que pertenecen a áreas lingüísticas donde se profundiza en el dominio de un idioma.

Figura 3. Medias estimadas del ICoDD en español y el ICoDD en lengua extranjera.

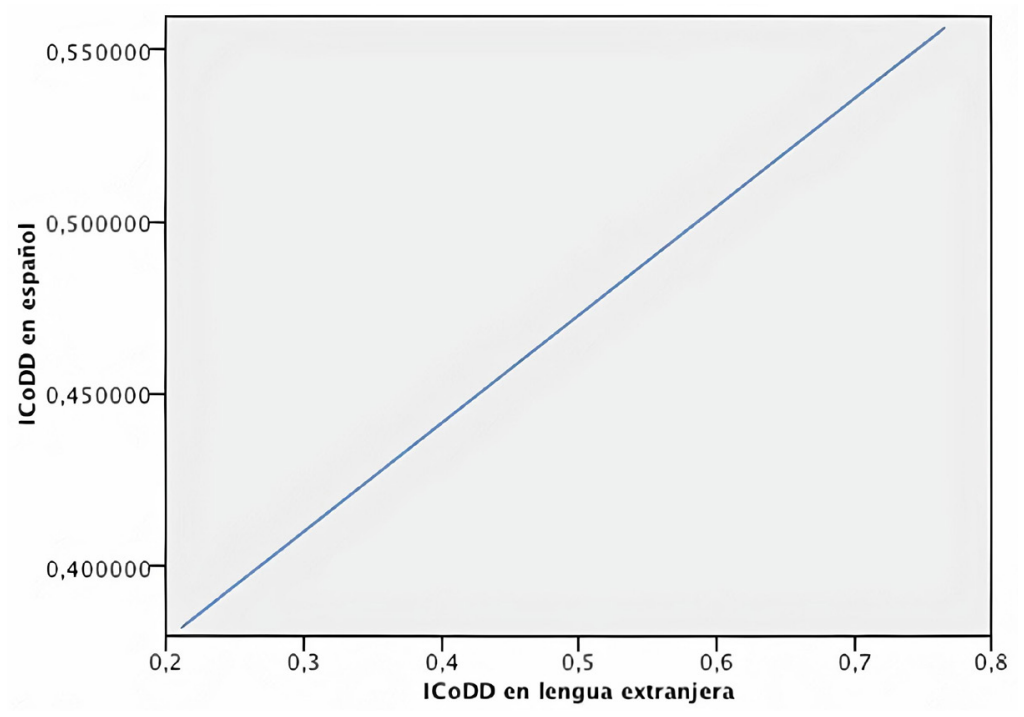

En cuanto a la especialidad del máster, en la Figura 4 se muestra la relación entre estas y el ICoDD en español agrupando los resultados en tres subconjuntos. El primero corresponde a la especialidad de Matemáticas, la cual se sitúa en el mínimo siendo, por tanto, la especialidad que incide negativamente en el ICoDD en español con una media de 0,4119 .

A continuación, le sigue el subconjunto que influye positivamente por ser el que presenta mayor ICoDD. Está formado por las especialidades de Lengua Extranjera (Inglés y Francés), Lengua Castellana y Literatura, Latín y Griego y Música, donde la media estimada es de 0,5107 . Por último, se presentan los datos del grupo formado por las especialidades de Geografía e Historia, Dibujo, Orientación, FOL, Hostelería y Turismo y TIPI. La media de este subconjunto es de 0,4645 y no presenta una influencia significativa ni positiva ni negativa. 
Figura 4. Medias estimadas del ICoDD en español y la especialidad del máster.

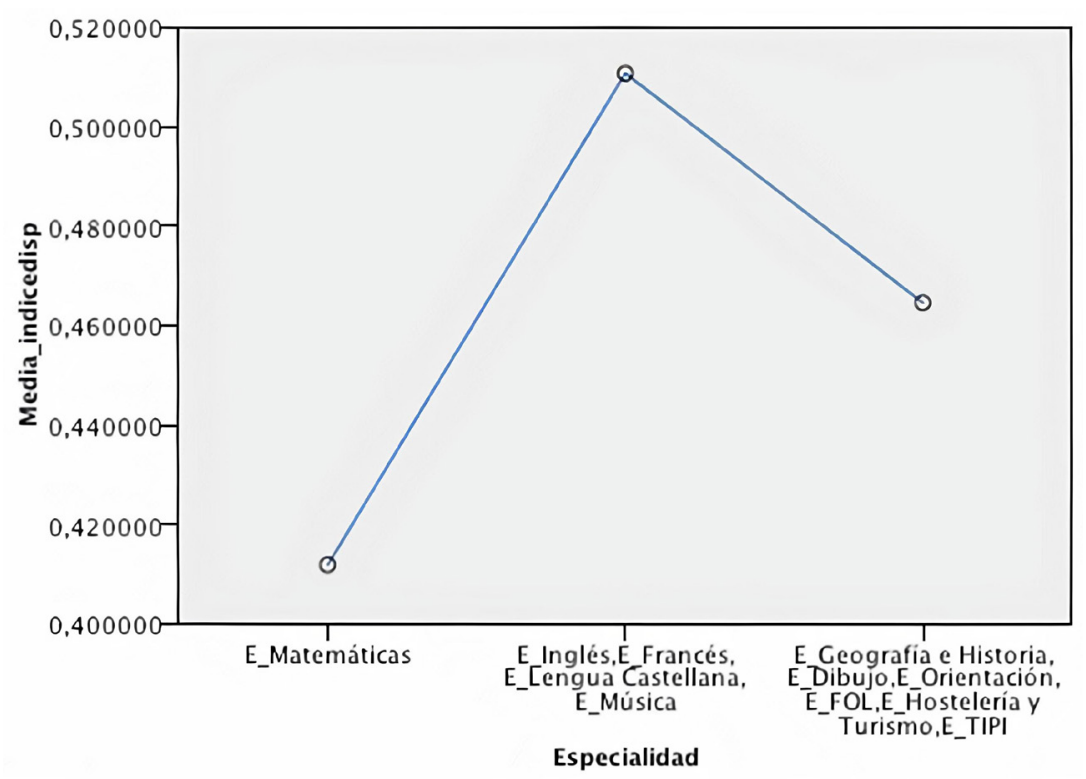

En cuanto al léxico especializado en español, la Figura 5 muestra la relación positiva entre el ICoDD y el ICIT en español. La incidencia de esta variable se justifica por diversas razones. En primer lugar, porque la propia naturaleza del ICoDD tiene en cuenta el vocabulario descentralizado y, por tanto, podría ser especializado. En segundo lugar, porque el hecho de que un informante posea mayor vocabulario quiere decir que también tiene un mejor dominio del lenguaje especializado, hallazgos coincidentes con investigaciones precedentes (Gómez Parra y Guerra Salas 2004; Hernández Muñoz 2015; Guerrero Ramos y Pérez Lagos 2018). 
Figura 5. Medias estimadas del ICoDD y el ICIT en español.

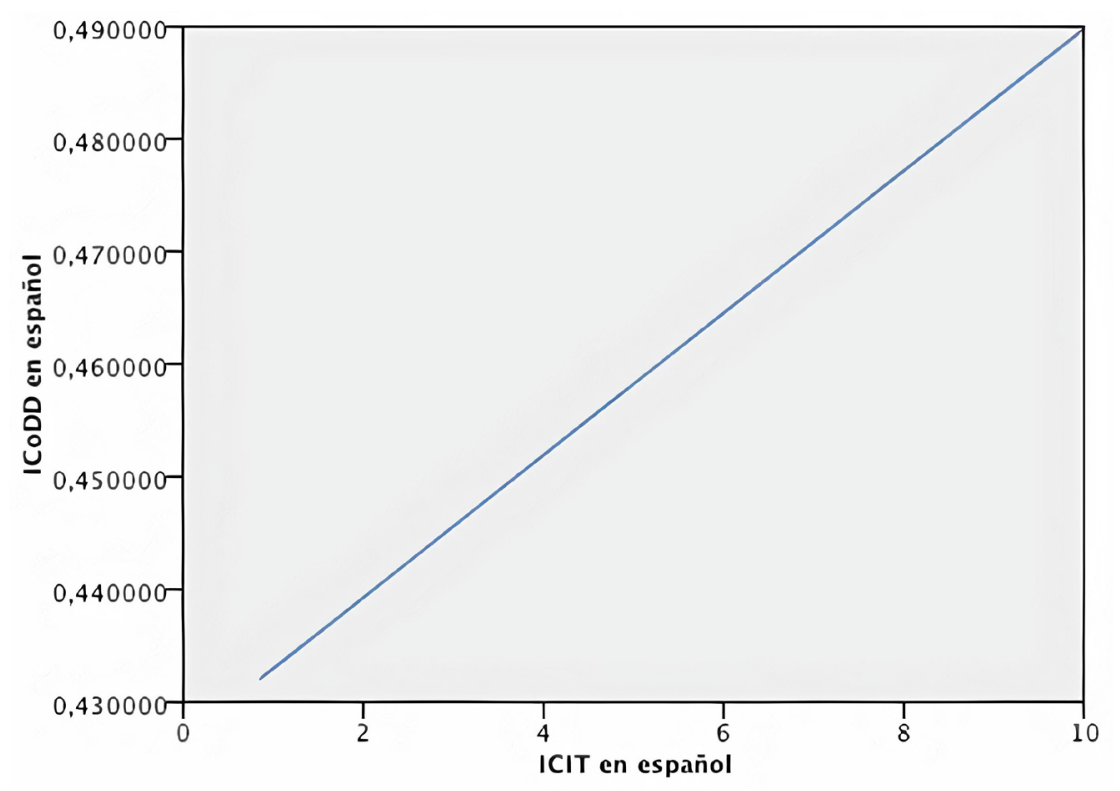

\section{CONCLUSIONES Y DiscuSIÓN}

Con esta investigación hemos pretendido avanzar en los estudios de disponibilidad léxica. En lo que respecta a los informantes, hemos tomado como referencia una muestra poblacional -el futuro profesorado de Educación Secundaria, Bachillerato, Formación Profesional y Escuelas oficiales de idiomas- aún poco explorada desde esta línea de investigación pero que, como se ha constatado, puede resultar de una enorme utilidad a la hora de plantear soluciones educativas de cara a mejorar la formación inicial del profesorado.

Para determinar cuáles son las variables más relacionadas con la competencia léxica de los futuros docentes hemos realizado un análisis de regresión múltiple. Asimismo, en lugar de tomar como referencia el número total de palabras para analizar la variable del léxico disponible, se ha tomado como referencia un índice novedoso en este ámbito de investigación, el ICoDD, que permite acceder mediante un riguroso tratamiento y análisis de los datos (tanto de corte cualitativo como cuantitativo) a la competencia léxica de cada informante, como se muestra en el trabajo de Ávila Muñoz, Sánchez Sáez y Odishelidze (2021). Los resultados han mostrado que los predictores en la competencia léxica de los futuros docentes de este estudio son: el ICoDD en lengua extranjera, la especialidad del máster, la capacidad de identificación de tecnicismos y los hábitos de lectura.

A este respecto, aunque en líneas generales exista consenso en la estrecha relación que guarda el hábito lector con la riqueza léxica (Qian 2002; Waring y Takaki 2003; Horst 
2005; Pigada y Schmitt 2006; Brown, Waring y Donkaewbua 2008; Riffo Ocares et al. 2014; Castillo Fadić y Sologuren Insúa 2020), no se trata de una línea muy explorada dentro de los estudios de disponibilidad léxica (Galloso Camacho 2004; Cepeda et al. 2017; Santos Díaz 2017b). Sin embargo, sus hallazgos son muy interesantes. De hecho, han demostrado que los estudiantes que leen más son capaces de reconocer más vocablos especializados (Riffo Ocares et al. 2014; Cepeda et al. 2017; Santos Díaz 2017b). Esta cuestión nos hace plantearnos la posibilidad de trazar, dentro de la formación inicial de docentes, itinerarios de lectura académica que, paulatinamente, vayan capacitando al profesorado para la utilización y reconocimiento del léxico especializado que posteriormente habrán de enseñar a sus estudiantes. Este tipo de actuaciones podría materializarse desde la existencia de Proyectos Lingüísticos de Centro (Pérez Invernón 2019) en las universidades, contemplando todo el ecosistema que rodea a las facultades de educación (Romero Oliva, Ambrós Pallarés y Trujillo Sáez 2020). de forma que se garanticen estrategias comunes tales como las propuestas por (Romero Oliva, Salvador Rosa y Trigo Ibáńez 2014 o Jarpa Azagra y Becerra Rojas 2019) en el ámbito de la escritura académica o se potencie la lectura académica (Santos Díaz, Juárez Calvillo y Trigo Ibánez 2021).

Enlazando con la idea anterior, es llamativo el hecho de que precisamente los informantes de especialidades no lingüísticas -futuros encargados de la docencia AICLE para la que se exige un nivel mínimo de B2- sean los que consignan una mayor pobreza léxica, lo que nos hace plantearnos ciertas dudas sobre su perfil como docente en la modalidad bilingüe. Es probable que estos estudiantes, al afrontar la labor docente, encuentren dificultades análogas a las planteadas por Pozuelo Estrada y Travé González (2019) y por Trigo Ibánez, Santos Díaz y Jiménez López (2021).

Estos hallazgos, si bien pudieran ser previsibles, también resultan preocupantes y más aún al tratarse de informantes procedentes de Andalucía, Comunidad Autónoma que ha apostado claramente por un modelo de enseñanza plurilingüe, manifiesto en su Plan Estratégico de Desarrollo de las Lenguas en Andalucía (2016). Es esperable que un importante número de nuestros estudiantes desempeñe su labor como docente de área no lingüística desde un modelo AICLE y, por ello, debe estar preparado para afrontar los retos profesionales. De acuerdo con la consultora Mckinsey (Barber y Mourshed 2008), el profesorado es uno de los pilares sobre el que descansa el éxito del sistema educativo y, por ende, la formación inicial debe aunar esfuerzos para lograr el máximo rendimiento. De nuevo se hace necesario tomar decisiones desde las políticas de formación docente, pues nuestros informantes serán -una vez insertados en el mercado laboral- los encargados de ejercer como modelo lingüístico a varias generaciones ya desarrollen la docencia de áreas lingüísticas como no lingüísticas (Romero Oliva y Trigo Ibánez 2018).

Indudablemente nuestro estudio se adscribe al contexto de la Universidad de Málaga y sus resultados, si bien no pueden ser generalizados, pretenden servir como un punto de partida para la reflexión sobre el modelo de formación inicial de docentes y realizar un llamamiento a la comunidad investigadora para realizar investigaciones similares en otros contextos a fin de poder contrastar resultados y buscar soluciones conjuntas. 


\section{OBRAS CITADAS}

Albarrán Lozano, Irene, Estany Montserrat Guillén y Mercedes Ayuso Gutiérrez. 2003. "Modelo de regresión lineal múltiple y modelos de elección discreta (logit, probit, multinomiales y censurado)”. En Jean Pierre Lévy Mangin y Jesús Varela Mallou (Coord.), Análisis Multivariable para las Ciencias Sociales. Madrid: Prentice Hall. 277-326.

Ávila Muñoz, Antonio Manuel y José María Sánchez Sáez. 2010. "La disponibilidad léxica. Antecedentes y fundamentos". En Antonio Manuel Ávila Muñoz y Juan Andrés Villena Ponsoda (Eds.), Variación social del léxico disponible en la ciudad de Málaga. Málaga: Editorial Sarriá.

. 2014. "Fuzzy sets and Prototype Theory: Representational model of cognitive community structures based on lexical availability trials". Review of Cognitive Linguistics 12.1: 133-159. doi: 10.1075/rcl.12.1.05avi

Ávila Muñoz, Antonio Manuel, José María Sánchez Sáez y Nana Odishelidze. 2021. "Dispocen. Mucho más que un programa para el cálculo de la disponibilidad léxica”. ELUA, 35: 9-36. doi: 10.14198/ELUA2021.35.1

Ávila Muñoz, Antonio Manuel y Juan Andrés Villena Ponsoda (Eds.). 2010. Variación social del léxico disponible en la ciudad de Málaga. Málaga: Editorial Sarriá. . 2011. Estudios sobre el español de Málaga. Pronunciación, vocabulario y sintaxis. Málaga: Editorial Sarriá.

Ávila Muñoz, Antonio Manuel, Inmaculada Clotilde Santos Díaz y Ester Trigo Ibáñez. 2020. "Análisis léxico-cognitivo de la influencia de los medios de comunicación en las percepciones de universitarios españoles ante la COVID-19”. Círculo De Lingüística Aplicada a La Comunicación, 84: 85-95. doi: 10.5209/clac.70701

Barber, Michael y Mona Mourshed. 2008. Cómo hicieron los sistemas educativos con mayor desempeño del mundo para alcanzar sus objetivos. Buenos Aires: Mckinsey y Company.

Brown, Ronan, Rob Waring y Sangrawee Donkaewbua. 2008. "Incidental vocabulary acquisition from reading, reading-while-listening, and listening to stories". Reading in a Foreign Language 20.2: 136-163.

Callealta Barroso, Francisco Javier y Diego Javier Gallego Gallego. 2016. "Measures of lexical availability: comparability and standardization”. Boletín de filología 51.1: 39-92. doi: 10.4067/S0718-93032016000100002

Calvo Gómez, Félix. 1993. Técnicas estadisticas multivariantes: con resolución de ejercicios prácticos mediante los paquetes estadísticos SPSS y PROGSTAD. País Vasco: Universidad de Deusto.

Castillo Fadić, María Natalia y Enrique Sologuren Insúa. 2020. "Léxico frecuente, riqueza léxica y estereotipos sobre la lectura de profesores en formación”. Logos: Revista de Lingüistica, Filosofía y Literatura 30.1: 69-85. doi: 10.15443/RL3006

Cepeda, Milko, Ángela Cárdenas, Macarena Carrasco, Nicole Castillo, Flores, Joselyne Constanza González y Melanie Oróstica. 2017. "Relación entre disponibilidad léxica 
y comprensión lectora, en un contexto de educación técnico profesional rural”. Revista Sophia Austral 1.1: 81-93. <http://www. sophiaaustral.cl/index.php/shopiaaustral/ article/view/51/46>

Cerda Etchepare, Gamal, Pedro Salcedo Lagos, Carlos Pérez Wilson y Verónica Marín Díaz. 2017. "Futuros Profesores de Matemáticas: Rol de la Disponibilidad Léxica, Esquemas de Razonamiento Formal en Logros Académicos Durante su Formación Inicial”. Formación universitaria 10.1: 33-46. doi: 10.4067/S0718-50062017000100005

De la Maya Retamar, Guadalupe. 2015. El desarrollo de la competencia léxica en la adquisición del francés como segunda lengua extranjera en la ESO. Badajoz: Universidad de Extremadura. Tesis doctoral.

De la Maya Retamar, Guadalupe y Magdalena López Pérez. 2019. Disponibilidad léxica en lengua extranjera ingles de futuros docentes de Educación Primaria. En María Ángeles Calero Fernández (Ed.) II Jornadas de investigación en disponibilidad léxica. Libro de resúmenes. Lleida: Universitat de Lleida. 11-15.

2020. "Disponibilidad léxica en inglés de futuros profesores de Educación Primaria". Tejuelo 32: 359-390. doi: 10.17398/1988-8430.32.359

. 2021. "Hábitos lectores y competencia léxica de futuros profesores de inglés en Educación Primaria”. Educação \& Formação, 6.1: 1-21. doi: 10.25053/redufor. v6i1.3504

De la Maya Retamar, Guadalupe y Mora Ramos, Inmaculada. 2019. "Estudio del conocimiento léxico en FLE de estudiantes españoles de Secundaria”. Revista Complutense de Educación 30.2: 527-543. doi: 10.5209/RCED.57773

Dörnyei, Zoltán y Kata Csizér. 2012. "How to design and analyze surveys in SLA research?" En Alison Mackey y Susan M. Gass (Eds.), Research methods in second language acquisition: A practical guide. Malden, MA: Wiley-Blackwell. 74-94.

Ferreira, Roberto. 2006. Disponibilidad léxica en inglés como lengua materna e inglés como lengua extranjera. Estudio del léxico disponible desde un enfoque psicolingüistico. (Tesis de magíster inédita). Universidad de Concepción.

Ferreira, Roberto y Max Sergio Echeverría. 2010-2011. "Redes semánticas en el léxico disponible de inglés L1 e inglés LE”. Onomázein 21: 133-153.

Ferreira, Roberto, Jaime Garrido Moscoso y Alexia Guerra Rivero. 2019. "Predictors of lexical availability in English as a second language". Onomázein 46: 18-34. doi: 10.7764/onomazein.46.03

Galloso Camacho, María Victoria. 2004. “Déficit léxico y comprensión lectora”. Puertas a la Lectura 17: 35-38.

Galloso Camacho, María Victoria y Montemayor Martín Camacho 2019. "La disponibilidad léxica en el Diccionario de onubensismos para la enseñanza de ELE”. Ogigia. Revista electrónica de estudios hispánicos 25: 211-231. doi: 10.24197/ogigia.25.2019.211-231

Gómez Parra, María Elena y Luis Guerra Salas (2004). Disponibilidad y fines específicos: análisis del centro de interés prensa. En Actas del III Congreso Internacional de la Aso- 
ciación Europea de Lenguas Para Fines Específicos. Granada: Universidad de Granada. 695-703.

Gougenheim, Georges, René Michéa, Paul Rivenc y Aurélien Sauvageot. 1956. L'élaboration du francais élémentaire. Paris: Didier.

. 1964. L'élaboration du francais fondamental (I degré). Étude sur l'élaboration d'un vocabulaire et d'une grammaire de base. Paris: Didier.

Guerrero Ramos, Gloria y Manuel Fernando Pérez Lagos. 2018. "Vocabulario especializado en el léxico disponible de los hablantes malagueños". Tonos Digital 34: 1-37.

Hernández-Muñoz, Natividad. 2004. El léxico disponible de los estudiantes conquenses. Salamanca: Universidad de Salamanca.

. 2015. "La evaluación de la competencia léxica adulta: una aproximación a través de la disponibilidad léxica y la especialización académica en preuniversitarios". $R e-$ vista de Filología 33: 79-99.

Hernández Muñoz, Natividad y Carmela Tomé Cornejo. 2017. "Léxico disponible en primera y segunda lengua: bases cognitivas". En Florencio del Barrio (Ed.). Palabras Vocabulario Léxico. La lexicología aplicada a la didáctica y a la diacronía. Venecia: Edizioni Ca’ Foscari. 99-122.

Herranz Llácer, Cristina Victoria. 2018. "Disponibilidad léxica de los futuros profesores de Educación Infantil y Primaria”. Revista Electrónica Interuniversitaria de Formación del Profesorado 21.1: 143-159. doi: 10.6018/ reifop.21.1.295271 . 2019. "El estado actual de los estudios de disponibilidad léxica en España". Lingüistica Española Actual XLI.1: 93-130.

Herranz Llácer, Cristina Victoria y Miguel Ángel Marcos Calvo. 2019. "Análisis del léxico disponible español de extranjeros que estudian los grados de educación". Ogigia. Revista electrónica de estudios hispánicos 26: 5-30. doi: 10.24197/ogigia.26.2019.5-30

Hidalgo Gallardo, Matías. 2019. "El perfil léxico del manual de ELE Español Moderno 1 desde la óptica de la disponibilidad léxica: posibles mejoras para la selección del vocabulario". Ogigia. Revista electrónica de estudios hispánicos 25: 233-257. doi: 10.24197/ogigia.25.2019.233-257

Horst, Marlise. 2005. "Learning L2 vocabulary through extensive reading: A measurement study". The Canadian Modern Language Review 61: 355-382.

Jarpa Azagra, Marcela Vanesa y Becerra Rojas, Nelson. 2019. "Escritura para la reflexión pedagógica: concepciones y géneros discursivos que escriben los estudiantes en dos carreras de pedagogía”. Logos: Revista de Lingüistica, Filología y Literatura 29.2: 364381. doi: $10.15443 /$ RL2928

Jiménez Catalán, Rosa María (Ed.). 2014. Lexical Availability in English and Spanish as a Second Language. Dordrech: Springer Netherlands.

Juárez Calvillo, María. 2019. "Influencia de la formación inicial del profesorado en los hábitos lectores y en el concepto de educación literaria". Investigaciones Sobre Lectura 12: 99-115. doi: 10.37132/isl.v0i12.287

Junta de Andalucía. 2016. Plan Estratégico de Desarrollo de las Lenguas en Andalucía. Sevilla. 
López Chávez, Juan y Carlos Strassburger Frías. 1987. “Otro cálculo del índice de disponibilidad léxica. Presente y perspectivas de la investigación computacional en México". Actas del IV Simposio de la Asociación Mexicana de Lingüistica Aplicada. México: Universidad Nacional Autónoma de México.

López González, Antonio María. 2014. Disponibilidad léxica. Teoría método y análisis. Lodz: Universidad de Lodz.

López Morales, Humberto. 2011. "Una palabra inicial”. En Ester Trigo. Dialectología y Cultura: el léxico disponible de los preuniversitarios sevillanos. Valencia: Aduana Vieja. 15-21.

López Morales, Humberto y Ester Trigo Ibáńez. 2019. "La disponibilidad léxica: tendencias actuales y perspectivas de futuro". Ogigia. Revista electrónica de estudios hispánicos 25: 7-10. doi: 10.24197/ogigia.25.2019.7-10

Mackey, William. 1971. Le vocabulaire disponible du français. París-Bruselas-Montreal: Didier. Marcos Calvo, Miguel Ángel y Cristina Victoria Herranz Llácer. 2019. Compatibilidad de los léxicos disponibles de estudiantes de los cursos primero y cuarto de los grados de educación. En María Ángeles Calero Fernández (Ed.) II Jornadas de investigación en disponibilidad léxica. Libro de resúmenes. Lleida: Universitat de Lleida. 11-15.

Michéa, R. 1953. "Mots fréquents et mots disponibles. Un aspect nouveau de la statistique du langage". Les Langues Modernes 47: 338-344.

Mora Ramos, Inmaculada. 2014. Evaluación de la competencia léxica en lenguas extranjeras: análisis del tamaño de vocabulario receptivo en alumnos AICLE y no AICLE de 60 de Primaria (Trabajo Fin de Máster inédito). Badajoz: Universidad de Extremadura.

Moreno Fernández, Francisco, José Enrique Moreno Fernández y Antonio García de las Heras. 1995. "Cálculo de disponibilidad léxica. El programa LexiDisp". Lingüistica 7: 243-249.

Paredes García, Florentino. 1999. "La ortografía en las encuestas de disponibilidad léxica". Revista Estudio Adquisición de la Lengua Española (REALE) 11: 75-98. . 2012. "Desarrollos teóricos y metodológicos recientes de los estudios de disponibilidad léxica”. Revista Nebrija de Lingüistica Aplicada 11. <https://www.nebrija.com/ revista-linguistica/files/articulosPDF/articulo_5285dd9dbe4d3.pdf>

Pérez Invernón, América. 2019. "El Proyecto Lingüístico de Centro: una evolución necesaria para la mejora de la Competencia en Comunicación Lingüística”. Tejuelo 30: 13-36. doi: 10.17398/1988-8430.30.13

Pigada, Maria y Norbert Schmitt. 2006. "Vocabulary acquisition from extensive reading: A case study". Reading in a Foreign Language 18.1: 1-28.

Pozuelo Estrada, Francisco José y Gabriel Travé González. 2019. "El trabajo por proyectos como alternativa metodológica para la integración lingüística y curricular. Estudio de un caso en un centro bilingüe". Profesorado. Revista de Curriculum y Formación de Profesorado 23.1: 421-440. doi:10.30827/profesorado.v23i1.9161

Qian, David. 2002. "Investigating the relationship between vocabulary knowledge and academic reading performance: an assessment perspective". Language Learning 52.3: 513-536. 
Quintanilla Espinoza, Angie y Pedro Salcedo Lagos. 2019. "Disponibilidad léxica en procesos de formación inicial de futuros profesores de inglés". Revista brasilera de lingüistica aplicada 19.3: 529-544. doi: 10.1590/1984-6398201913157

Riffo Ocares, Bernardo, Fernando Reyes Reyes, Abraham Novoa Lagos, Mónica Véliz de Vos y Ginette Castro Yánez. 2014. "Competencia léxica, comprensión lectora y rendimiento académico en estudiantes de enseñanza media”. Literatura y lingüistica 30: 165-180. doi: 10.4067/S0716-58112014000200009

Rodríguez Gómez, Gregorio, Javier Gil Flores y Eduardo García Jiménez. 1996. Metodología de la investigación cualitativa. Málaga: Ediciones Algibe.

Rojas Díaz, Darío Fernando, Carolina Zambrano Matamala y Pedro Salcedo Lagos. 2017. "Metodología de Análisis de Disponibilidad Léxica en Estudiantes de Pedagogía a Través de la Comparación Jerárquica de Lexicones". Formación Universitaria 10.4: 3-14. doi:10.4067/S0718-50062017000400002. . 2019. "Método para la formación de grupos colaborativos mediante disponibilidad léxica". Revista Electrónica de Investigación Educativa 21-e36: 1-12. doi:10.24320/ redie.2019.21.e36.2095

Romero Oliva, Manuel Francisco, Alba Ambrós Pallarés y Fernando Trujillo Sáez. 2020. "Hábitos lectores de los adolescentes en un ecosistema llamado escuela: factores determinantes en estudiantes de educación secundaria”. Investigaciones Sobre Lectura 13: 18-34. doi: 0.37132/isl.v0i13.295.

Romero Oliva, Manuel Francisco, Aurora Salvador Rosa y Ester Trigo Ibáńez. 2014. "Propuestas para trabajar la escritura académica en los estudios universitarios". En Manuel Francisco Romero Oliva (Coord.). La escritura académica: diagnóstico y propuestas de actuación. Barcelona: Octaedro. 87-100.

Romero Oliva, Manuel Francisco y Ester Trigo Ibáńez. 2018. "Los proyectos lingüísticos de centro. Desarrollar la comprensión lectora en áreas no lingüísticas". Textos. Didáctica de la Lengua y la Literatura 79: 51-59.

Rubio Lastra, Miguel. 2020. "Evaluación de la suficiencia léxica de estudiantes taiwaneses universitarios de ELE A1 por medio de la disponibilidad léxica y el Plan curricular del Instituto Cervantes". Lingüistica en la red XVII: 1-42.

Samper Padilla, José Antonio. 1998. "Criterios de edición del léxico disponible: sugerencias”. Lingüistica 10: 311-33.

Samper Padilla, José Antonio, Juan José Bellón Fernández y Marta Samper Hernández 2003. "Proyecto de estudio de la disponibilidad léxica en español". En Raúl Ávila, José Antonio Samper, Héctor Ueda et al., Pautas y pistas en el análisis del léxico hispano (americano), MadridFrankfurt: IberoamericanaVervuert. 27-141.

Sánchez-Saus Laserna, Marta. 2016. Léxico disponible de los estudiantes de español como lengua extranjera en las universidades andaluzas. Sevilla: Universidad de Sevilla.

Santos Díaz, Inmaculada Clotilde. 2017a. "Selección del léxico disponible: propuesta metodológica con fines didácticos”. Porta Linguarum. Revista internacional de didáctica de las lenguas extranjeras 27: 122-139. 
. 2017b. "Incidencia de la lectura en el vocabulario en lengua materna y extranjera". Ocnos 16.1: 79-88. doi: 10.18239/ocnos_2017.16.1.1151

. 2017c. "Organización de las palabras en la mente en lengua materna y lengua extranjera (inglés y francés)". Pragmalingüistica 25: 603-617.

. 2018. "El efecto de la clase social en la competencia léxica de estudiantes de postgrado". e-AESLA 4: 315-322 <https://cvc.cervantes.es/lengua/eaesla/pdf/04/31.pdf> . 2020. El léxico bilingüe del futuro profesorado. Análisis y pautas para estudios de disponibilidad. Berlín: Peter Lang.

Santos Díaz, Inmaculada Clotilde, María Juárez Calvillo y Ester Trigo Ibáñez. 2021. "Motivación por la lectura académica de futuros docentes”. Educação \& Formação, 6.1: 1-21. doi:10.25053/redufor.v6i1.3535

Santos Díaz, Inmaculada Clotilde, Ester Trigo Ibáñez y Manuel Francisco Romero Oliva. 2019. "Análisis comparativos e intermuestrales de los errores ortográficos en estudios de disponibilidad léxica en español y en lengua extranjera”. Lingüistica Española Actual XLI.1: 131-158.

. 2020. "La activación del léxico disponible y su aplicación a la enseñanza de lenguas". Porta Linguarum. Revista internacional de didáctica de las lenguas extranjeras 33: 75-93.

Trigo Ibáñez, Ester, Manuel Francisco Romero Oliva e Inmaculada Clotilde Santos Díaz. 2018. "Elaboración de un corpus cacográfico desde la disponibilidad léxica en estudiantes sevillanos. Un análisis para la enseñanza de la lengua”. Revista de Lingüística y Lenguas Aplicadas 13: 119-131. doi: 10.4995/rlyla.2018.9176

. 2019a. "Aproximación empírica desde la disponibilidad léxica a la influencia de los factores sociolingüísticos en el dominio ortográfico". Cultura y Educación 31.4: 814-844. doi: 10.1080/11356405.2019.1659007

. 2019b. "Aproximación al léxico gastronómico dialectal andaluz desde los repertorios de disponibilidad léxica para una propuesta didáctica". Verba Hispánica XXVII: 115-130. doi:10.4312/vh.27.1.115-130

. 2020. "Disponibilidad léxica y dominio de la ortografía: un estudio empírico basado en la influencia de los factores sociales”. Onomázein 47: 27-45. doi: 10.7764/ onomazein. 47.02

Trigo Ibáñez, Ester, Inmaculada Clotilde Santos Díaz y Guillermina Jiménez López. 2021. "Comunicarse en la escuela plurilingüe: la formación en lengua extranjera del futuro profesorado”. Revista Latina De Comunicación Social 79: 53-75. doi: 10.4185/ RLCS-2021-1497

Trigo Ibáñez, Ester, Inmaculada Clotilde Santos Díaz y Manuel Francisco Romero Oliva. 2021. Recopilación del léxico disponible dialectal en Andalucía sobre alimentos desde el análisis sociolingüístico de la muestra sevillana. Revista Española de Lingüistica Aplicada 34.1: 284-315.

Valenzuela Castellanos, Marco, María Victoria Pérez Villalobos, Claudio Bustos y Pedro Salcedo Lagos. 2018. "Cambios en el concepto aprendizaje de estudiantes de peda- 
gogía: análisis de disponibilidad léxica y grafos". Estudios Filológicos 61: 143-173. doi:10.4067/S0071-17132018000100143

Waring, Rob y Misako Takaki. 2003. "At what rate do learners learn and retain new vocabulary from reading a graded reader?". Reading in a Foreign Language 15: 130-163.

Zambrano Matamala, Carolina, Rojas Díaz, Darío Fernando y Pedro Salcedo Lagos. 2019. "Revisión Sistemática a Estudios de Disponibilidad Léxica en la Base de Datos Scielo y sus Aportes a Educación”. Información Tecnológica 30.4: 189-198. doi: 10.4067/ S0718-07642019000400189 
Anexo. Prueba de identificación del léxico especializado

Sitúe en cada recuadro el Centro de Interés (1-9) en el que cree que corresponde cada palabra. Por ejemplo, tórax $=1$ (partes del cuerpo). Si no sabe la respuesta deje el recuadro en blanco.

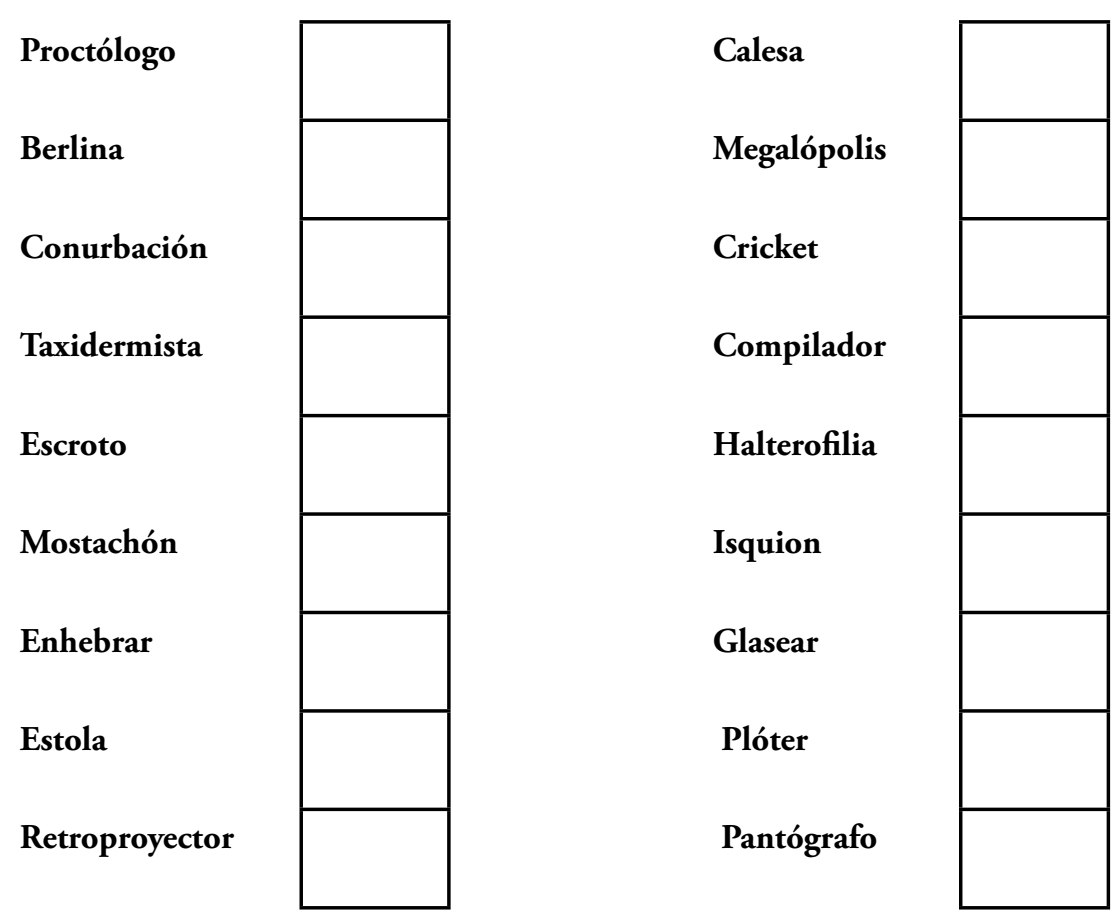

Fuente: Santos Díaz (2020: 226)

Centros de inTERÉs

1. Partes del cuerpo

2. La ropa

3. Comidas y bebidas

4. La escuela (muebles y materiales)

5. La ciudad

6. Medios de transporte

7. Juegos y distracciones

8. Profesiones y oficios

9. Ordenadores e internet 
